Proyecciones Journal of Mathematics

Vol. 37, No 4, pp. 603-612, December 2018.

Universidad Católica del Norte

Antofagasta - Chile

\title{
On some difference sequence spaces of interval numbers
}

\author{
Binod Chandra Tripathy \\ Tripura University, India \\ Shyamal Debnath \\ Tripura University, India \\ and \\ Subrata Saha \\ Tripura University, India \\ Received : December 2017. Accepted : February 2018
}

\begin{abstract}
In this paper we introduce the sequence spaces $c_{0}{ }^{i}(\Delta), c^{i}(\Delta)$ and $\ell_{\infty}^{i}(\Delta)$ of interval numbers and study some of their algebraic and topological properties. Also we investigate some inclusion relations related to these spaces.
\end{abstract}

Keywords : Sequence space; interval numbers; difference operator; solidity.

2000 Mathematics Subject Classification : 40C05; 40J05; 46A45 


\section{Introduction}

Many mathematical structures have been constructed with real or complex numbers. In recent years, these mathematical structures were replaced by fuzzy numbers and interval numbers. These are very popular since 1965 due to the introduction of fuzzy sets by L.A.Zadeh. The notion of fuzzy real numbers has been applied for introducing converging sequences of fuzzy numbers by Tripathy and Debnath [6], Tripathy and Baruah [4], Tripathy and Sen [5] and many others. Furthermore, these mathematical structures were replaced by intuitionistic fuzzy numbers or two dimensional interval numbers. Interval arithmetic was first suggested by Dwyer [12] in 1951. Development of interval arithmetic as a formal system and evidence of its value as a computational device was provided by Moore [15] in 1959 and Moore and Yang [16] in 1962. Chiao [8] introduced sequence of interval numbers and defined usual convergence of sequences of interval numbers. Sengonul and Eryilamz [10] introduced and studied bounded and convergent sequences of interval numbers and established that these spaces are complete metric spaces. Esi $[1,2]$ studied the lacunary single and double sequence spaces of interval numbers. Dutta [3] studied different properties of p-absolutely summable sequences of interval numbers. The notion of diference sequence spaces was first introduced by Kizmaz [7] and this concept was generalized by Et and Colak [9],Tripathy et.al. [4, 6] in different ways. These were further studied by Debnath et.al. [20, 21, 22] and many others.

\section{Preliminaries:}

An interval number $\bar{x}$ is a closed subset of the real numbers and denoted by $\bar{x}=\left[x_{l}, x_{u}\right]$, where $x_{l} \leq x_{u}$ and $x_{l}, x_{u}$ both are real numbers. The set all real valued closed intervals is denoted by $R(I)$. The absolute value (magnitude or interval norm) of an interval number is defined by

$$
|\bar{x}|=\max \left\{\left|x_{l}\right|,\left|x_{u}\right|\right\} .
$$

In general, the distributive laws do not hold for interval arithmetic. If $\bar{x}, \bar{y}, \bar{z}$ are any three intervals then it is easy to verify,

$$
\bar{x} \cdot(\bar{y}+\bar{z}) \subseteq \bar{x} \cdot \bar{y}+\bar{x} \cdot \bar{z}
$$




$$
\begin{gathered}
|\bar{x} \cdot(\bar{y}+\bar{z})| \leq|\bar{x} \cdot \bar{y}|+|\bar{x} \cdot \bar{z}| \\
|\bar{x} \cdot \bar{y}| \leq|\bar{x}| \cdot|\bar{y}|
\end{gathered}
$$

The set of all interval numbers $R(I)$, is a complete metric space(One may refer to [10]) with the metric $d(\bar{x}, \bar{y})=\max \left\{\left|x_{l}-y_{l}\right|,\left|x_{r}-y_{r}\right|\right\}$.

Here after any sequence $\left(x_{k}\right)$ of interval numbers will be an element of $(R(I), d)$.

Definition 2.1[8]: A sequence $\left(\bar{x}_{k}\right)$ is said to be convergent to the interval number $\left(\bar{x}_{0}\right)$ if for a given $\varepsilon>0$ there exists a positive integer $n_{0}$ such that $d\left(\bar{x}_{k}, \bar{x}_{0}\right)<\varepsilon$ for all $k \geq n_{0}$, and denoted by $\lim _{k \rightarrow \infty} \bar{x}_{k}=\bar{x}_{0}$.

We denote the set of all sequences of interval number with real terms by $w^{i}$. Given two sequences of interval numbers in $w^{i}$, say $\left(\bar{x}_{k}\right)$ and $\left(\bar{y}_{k}\right)$, the linear structure of $w^{i}$ includes the addition of $\left(\bar{x}_{k}\right)+\left(\bar{y}_{k}\right)=\left[x_{k l}+y_{k l}, x_{k u}+\right.$ $\left.y_{k u}\right]$ and scalar multiplication $\alpha\left(\bar{x}_{k}\right)$ defined by $\alpha\left(\bar{x}_{k}\right)=\left[\alpha x_{k l}, \alpha x_{k u}\right]$, if $\alpha \geq 0$ and $\alpha\left(\bar{x}_{k}\right)=\left[\alpha x_{k u}, \alpha x_{k l}\right]$, if $\alpha \leq 0$.

Since the set of all intervals on $\mathrm{R}$ is a quasi-vector space, the set $w^{i}$ be regarded as a quasi vector space and the following rules are clearly satisfied: $\left(\bar{x}_{k}\right)+\left(\bar{y}_{k}\right)=\left(\bar{y}_{k}\right)+\left(\bar{x}_{k}\right) ;\left(\bar{x}_{k}\right)+\left(\left(\bar{y}_{k}\right)+\left(\bar{z}_{k}\right)\right)=\left(\left(\bar{x}_{k}\right)+\left(\bar{y}_{k}\right)\right)+\left(\bar{z}_{k}\right)$; $\left(\bar{x}_{k}\right)+\left(\bar{y}_{k}\right)=\left(\bar{x}_{k}\right)+\left(\bar{z}_{k}\right)$ implies $\left(\bar{y}_{k}\right)=\left(\bar{z}_{k}\right) ; \alpha\left(\left(\bar{x}_{k}\right)+\left(\bar{y}_{k}\right)\right)=\alpha\left(\bar{x}_{k}\right)+\alpha\left(\bar{y}_{k}\right)$ $;(\alpha+\beta)\left(\bar{x}_{k}\right)=\alpha\left(\bar{x}_{k}\right)+\beta\left(\bar{x}_{k}\right),($ where $\alpha, \beta>0) ; \alpha\left(\beta\left(\bar{x}_{k}\right)\right)=(\alpha \beta)\left(\bar{x}_{k}\right) ;$ $\left(\bar{x}_{k}\right)=[1,1]\left(\bar{x}_{k}\right)$. The zero element of $w^{i}$ is the sequence $\bar{\theta}=\left(\bar{\theta}_{k}\right)=([0,0])$, all terms of which are zero interval.

In [10], $c_{0}^{i}, c^{i}$ and $l_{\infty}^{i}$ denote the spaces of null, convergent and bounded sequences of interval numbers respectively, that is

$$
\begin{aligned}
& c_{0}^{i}=\left\{\bar{x}=\left(\bar{x}_{k}\right) \in w^{i}: \lim _{k \rightarrow \infty} \bar{x}_{k}=\theta, \text { where } \theta=[0,0]\right\} \\
& c^{i}=\left\{\bar{x}=\left(\bar{x}_{k}\right) \in w^{i}: \lim _{k \rightarrow \infty} \bar{x}_{k}=\bar{x}_{0}, \bar{x}_{0} \in R(I)\right\} \\
& \ell_{\infty}^{i}=\left\{\bar{x}=\left(\bar{x}_{k}\right) \in w^{i} \sup _{k}\left\{\left|x_{k l}\right|,\left|x_{k u}\right|\right\}<\infty\right\}
\end{aligned}
$$

The space $c_{0}^{i}$ (or $c^{i}$ or $\ell_{\infty}^{i}$ ) forms a complete metric space with the metric defined by 
$\widetilde{d}\left(\bar{x}_{k}, \bar{y}_{k}\right)=\sup _{k} \max \left\{\left|x_{k l}-y_{k l}\right|,\left|x_{k u}-y_{k u}\right|\right\}$ for all $(\bar{x}),(\bar{y}) \in c_{0}{ }^{i}$ (or $c^{i}$ or $\ell_{\infty}^{i}$ ) and are normed interval space with the norm $\|x\|=\underset{k}{\sup \max }$ $\left\{\left|x_{k l}\right|,\left|x_{k u}\right|\right\}$, where $\bar{x}=\left(\bar{x}_{k}\right) \in c_{0}^{i}$ (or $c^{i}$ or $\left.\ell_{\infty}^{i}\right)$.

Kizmaz [7] defined the difference sequences of real numbers by $X(\Delta)=\left\{x=\left(x_{k}\right) \in w:\left(\Delta x_{k}\right) \in X\right\}$, for $X=\ell_{\infty}, c$ and $c_{0}$, where $\Delta \mathrm{x}_{k}$ $=\left(x_{k}-x_{k+1}\right)$ The above spaces are complete normed spaces defined by the norm $\|x\|_{\triangle}=\left|x_{1}\right|+\underset{k}{\sup }\left|\Delta x_{k}\right|$

Definition 2.2: An interval valued sequence $\bar{x}=\left(\bar{x}_{k}\right)$ is said to be interval valued Cauchy sequence if for every $\varepsilon>0$ there exists a $k_{0} \in N$ such that $\bar{d}\left(\bar{x}_{n}, \bar{x}_{m}\right)<\varepsilon$ for all $n, m>k_{0}$.

Definition 2.3: Let $\lambda^{i}$ be a sequence space of interval numbers. Then $\lambda^{i}$ is called normal or solid if $\bar{y} \in \lambda^{i}$ whenever $\|\bar{y}\| \leq\|\bar{x}\|$, for some $\bar{x}=\left(\bar{x}_{k}\right) \in \lambda^{i}$.

Definition 2.4: A sequence space $\lambda^{i}$ is said to be a sequence algebra if $\left(\bar{x}_{k}\right)\left(\bar{y}_{k}\right) \in \lambda^{i}$ whenever $\left(\bar{x}_{k}\right) \in \lambda^{i},\left(\bar{y}_{k}\right) \in \lambda^{i}$.

Definition 2.5: A sequence space $\lambda^{i}$ is said to be convergence free if $\left(\bar{y}_{k}\right) \in \lambda^{i}$ whenever $\left(\bar{x}_{k}\right) \in \lambda^{i}$ and $\bar{x}_{k}=\bar{\theta}$ implies $\bar{y}_{k}=\bar{\theta}$.

Definition 2.6: A sequence space $\lambda^{i}$ is said to be a monotone if $\lambda^{i}$ contains the canonical pre-images of all its step spaces.

Remark 2.1: A sequence space is solid implies that it is monotone.(One may refer to Kamthan and Gupta [11])

\section{Main Results}

In this paper we consider the difference operator on the sequences of interval numbers defined by

$$
\Delta \bar{x}=\left(\Delta \bar{x}_{k}\right)=\left(\left[\Delta x_{k l}, \Delta x_{k u}\right]\right) .
$$

We introduce the classes of difference null, convergent and bounded sequences of interval numbers. We denote the difference sequences of null, 
convergent, bounded sequences of interval numbers by $c_{0}{ }^{i}(\Delta), c^{i}(\Delta)$ and $\ell_{\infty}^{i}(\Delta)$ respectively, defined by

$$
\begin{aligned}
& c_{0}^{i}(\Delta)=\left\{\bar{x}=\left(\bar{x}_{k}\right) \in w^{i}: \lim _{k} \Delta \bar{x}_{k}=\theta, \text { where } \theta=[0,0]\right\} . \\
& c^{i}(\Delta)=\left\{\bar{x}=\left(\bar{x}_{k}\right) \in w^{i}: \lim _{k} \Delta \bar{x}_{k}=\bar{x}_{k}, \bar{x}_{k} \in R(I)\right\} . \\
& \ell_{\infty}^{i}(\Delta)=\left\{\bar{x}=\left(\bar{x}_{k}\right) \in w^{i}: \sup _{k}\left\{\left|\Delta x_{k l}\right|,\left|\Delta x_{k u}\right|\right\}<\infty\right\} .
\end{aligned}
$$

It can be easily verified that the spaces $c_{0}{ }^{i}(\Delta), c^{i}(\Delta)$ and $\ell_{\infty}^{i}(\Delta)$ are subsets of the space $w^{i}$. Besides, for all $\left(\bar{x}_{k}\right),\left(\bar{y}_{k}\right) \in c_{0}{ }^{i}(\Delta)\left(\operatorname{or} c^{i}(\Delta), \ell_{\infty}^{i}(\Delta)\right.$ ) the distance $\widetilde{d}$ is defined by

$$
\widetilde{d}\left(\bar{x}_{k}, \bar{y}_{k}\right)=\sup _{k}\left\{\max \left\{\left|\Delta x_{k l}-\Delta y_{k l}\right|,\left|\Delta x_{k u}-\Delta y_{k u}\right|\right\}\right\}
$$

which satisfies all the axioms of a metric. Thus $\left(c_{0}{ }^{i}(\Delta), \tilde{d}\right),\left(c^{i}(\Delta), \tilde{d}\right)$ and $\left(\ell_{\infty}^{i}(\Delta), \widetilde{d}\right)$ are metric spaces.

Theorem 3.1: $c_{0}{ }^{i}(\Delta), c^{i}(\Delta)$ and $\ell_{\infty}^{i}(\Delta)$ are complete metric spaces with the metric defined by (3.1).

Proof: We prove the result for the class $c_{0}{ }^{i}(\Delta)$. The rest can be established similarly.

Let $\left(\bar{x}_{n}\right)$ be a Cauchy sequence. Then for each $\varepsilon>0$, there exists a $k_{0} \in N$ such that $\widetilde{d}\left(\bar{x}_{n}, \bar{x}_{m}\right)<\varepsilon$, whenever $n, m \geq n_{0}$, where $\bar{x}_{n}=\left(\bar{x}_{n}^{k}\right) \in$ $c_{0}^{i}(\Delta)$.

Hence $\sup _{k}\left\{\max \left\{\left|\Delta x_{n l}^{k}-\Delta x_{m l}^{k}\right|,\left|\Delta x_{n u}^{k}-\Delta x_{m u}^{k}\right|\right\}<\varepsilon\right.$.

Thus we have $\left|\Delta x_{n l}^{k}-\Delta x_{m l}^{k}\right|<\varepsilon$ and $\left|\Delta x_{n u}^{k}-\Delta x_{m u}^{k}\right|<\varepsilon$.

Let $k$ be fixed, then $\left(\Delta x_{n l}^{k}\right)$ and $\left(\Delta x_{n u}^{k}\right)$ are both Cauchy sequence of real numbers. Since the set of all real numbers is complete, so $\lim _{n \rightarrow \infty} \Delta x_{n l}^{k}=$ $l_{1}$ and $\lim _{n \rightarrow \infty} \Delta x_{n u}^{k}=l_{2}$

Taking $m \rightarrow \infty$, we have $\left|\Delta x_{n l}^{k}-l_{1}\right|<\varepsilon$ and $\left|\Delta x_{n u}^{k}-l_{2}\right|<\varepsilon, \forall n \geq n_{0}$ and $\forall k \in N$,

i.e, $\Delta x_{n l}^{k} \in\left(l_{1}-\varepsilon, l_{1}+\varepsilon\right)$ and $\Delta x_{n u}^{k} \in\left(l_{2}-\varepsilon, l_{2}+\varepsilon\right)$

So, $\max \left\{\left|\Delta x_{n l}^{k}\right|,\left|\Delta x_{n u}^{k}\right|\right\}<\max \left\{l_{1}+\varepsilon, l_{2}+\varepsilon\right\}$

i.e., $\sup _{k}\left\{\max \left\{\left|\Delta x_{n l}^{k}\right|,\left|\Delta x_{n u}^{k}\right|\right\}\right\}<\varepsilon^{\prime}$, where $\varepsilon^{\prime}=\max \left\{l_{1}+\varepsilon, l_{2}+\varepsilon\right\}$

i.e, $\widetilde{d}\left(\bar{x}_{n}, \theta\right)<\varepsilon^{\prime}, \forall n \geq n_{0}$

This implies that $\left(\bar{x}_{n}\right)$ is a convergent sequence and converge to $\theta \in$ $c_{0}^{i}(\Delta)$. This completes the proof. 
The norm function on the sequences of interval numbers can be extended to the difference sequence spaces of interval numbers, denoted by $\lambda^{i}(\Delta)$, which is a subset of $w^{i}$, where $\lambda^{i}(\Delta)=c_{0}^{i}(\Delta)$ or $c^{i}(\Delta)$ or $\ell_{\infty}^{i}(\Delta)$.

Definition 3.1. A norm on $\lambda^{i}(\Delta)$ is a non-negetive function $\|\cdot\|_{\lambda^{i}}=$ $\lambda^{i}(\Delta) \rightarrow R^{+} \cup\{0\}$ that satisfies the following properties: $\forall \bar{x}, \bar{y} \in \lambda^{i}(\Delta)$ and $\forall \alpha \in \mathrm{R}$,

$N_{1} \cdot\|\bar{x}\|_{\lambda^{i}}>0, \forall \bar{x} \in \lambda^{i}(\Delta)-\{\theta\}$

$N_{2} \cdot\|\bar{x}\|_{\lambda^{i}}=0 \Leftrightarrow \bar{x}=\theta$

$N_{3} \cdot\|\bar{x}+\bar{y}\|_{\lambda^{i}} \leq\|\bar{x}\|_{\lambda^{i}}+\|\bar{y}\|_{\lambda^{i}}$

$N_{4} \cdot\|\alpha \bar{x}\|_{\lambda^{i}}=|\alpha|\|\bar{x}\|_{\lambda^{i}}$

Theorem 3.2. The spaces $c_{0}^{i}(\Delta), c^{i}(\Delta)$ and $\ell_{\infty}^{i}(\Delta)$ are normed spaces with the norm

$$
\|\bar{x}\|_{\Delta}=\max \left(\left|x_{l}^{1}\right|,\left|x_{u}^{1}\right|\right)+\sup _{k} \max \left\{\left|\Delta x_{l}^{k}\right|,\left|\Delta x_{u}^{k}\right|\right\} \ldots
$$

Proof: Let $\lambda^{i}(\Delta)=c_{0}{ }^{i}(\Delta)$ or $c^{i}(\Delta)$ or $l_{\infty}^{i}(\Delta)$ and $\bar{x}, \bar{y} \in \lambda^{i}(\Delta)$.

$N_{1} \cdot\|\bar{x}\|_{\lambda^{i}}=\sup _{k} \max \left\{\left|\Delta x_{l}^{k}\right|,\left|\Delta x_{u}^{k}\right|\right\}$.

It can be easily verified that $\|\bar{x}\|_{\lambda^{i}}>0$ for $\bar{x} \in \lambda^{i}(\Delta)-\{\theta\}$

$N_{2} .\|\bar{x}\|_{\lambda^{i}}=0 \Leftrightarrow \max \left(\left|x_{l}^{1}\right|,\left|x_{u}^{1}\right|\right)+\sup _{k} \max \left\{\left|\Delta x_{l}^{k}\right|,\left|\Delta x_{u}^{k}\right|\right\}=0 \Leftrightarrow \bar{x}=$ $\theta$

$N_{3} .\|\bar{x}+\bar{y}\|_{\lambda^{i}}=\max \left(\left|x_{l}^{1}+y_{l}^{1}\right|,\left|x_{u}^{1}+y_{u}^{1}\right|\right)+\sup _{k} \max \left\{\left|\Delta\left(x_{l}^{k}+y_{l}^{k}\right)\right|, \mid \Delta\left(x_{u}^{k}+\right.\right.$ $\left.\left.y_{u}^{k}\right) \mid\right\}$

$\leq \max \left(\left|x_{l}^{1}\right|+\left|y_{l}^{1}\right|,\left|x_{u}^{1}\right|+\left|y_{u}^{1}\right|\right)+\sup _{k} \max \left\{\left|\Delta x_{l}^{k}\right|+\left|\Delta y_{l}^{k}\right|,\left|\Delta x_{u}^{k}\right|+\left|\Delta y_{u}^{k}\right|\right\}$

$=\max \left(\left|x_{l}^{1}\right|,\left|x_{l}^{u}\right|\right)+\sup _{k} \max \left\{\left(\left|\Delta x_{l}^{k}\right|,\left|\Delta x_{u}^{k}\right|\right)\right\}+\max \left(\left|y_{l}^{1}\right|,\left|y_{l}^{u}\right|\right)+\sup _{k}$ $\max \left\{\left(\left|\Delta y_{l}^{k}\right|,\left|\Delta y_{u}^{k}\right|\right)\right\}$

$=\|\bar{x}\|_{\lambda^{i}}+\|\bar{x}\|_{\lambda^{i}}$

$N_{4} \cdot\|\alpha \bar{x}\|_{\lambda^{i}}=\max \left(\left|\alpha x_{l}^{1}\right|,\left|\alpha x_{u}^{1}\right|\right)+\sup _{k} \max \left\{\left|\alpha \Delta x_{l}^{k}\right|,\left|\alpha \Delta x_{u}^{k}\right|\right\}$

$=\max \left(|\alpha|\left|x_{l}^{1}\right|,|\alpha|\left|x_{u}^{1}\right|\right)+\sup _{k} \max \left\{|\alpha|\left|\Delta x_{l}^{k}\right|,|\alpha|\left|\Delta x_{u}^{k}\right|\right\}$

$=|\alpha| \max \left(\left|x_{l}^{1}\right|,\left|x_{u}^{1}\right|\right)+|\alpha| \sup _{k} \max \left\{\left|\Delta x_{l}^{k}\right|,\left|\Delta x_{u}^{k}\right|\right\}$

$=|\alpha|\|\bar{x}\|_{\lambda^{i}}$

So $\|\bar{x}\|_{\lambda^{i}}$ is a norm on $\lambda^{i}(\Delta)$.

In view of the definition of norm on interval sequences and Theorem 3.1 , we formulate the following result. 
Theorem 3.3. The class of difference sequences of interval numbers $c_{0}{ }^{i}(\Delta), c^{i}(\Delta)$ and $\ell_{\infty}^{i}(\Delta)$ are Banach spaces with respect to the norm (3.2).

Theorem 3.4. The spaces $c_{0}{ }^{i}(\Delta), c^{i}(\Delta)$ and $\ell_{\infty}^{i}(\Delta)$ is neither solid nor monotone.

Example 3.1. Consider sequences $\bar{x}_{k}=[k, k]$, for all $k \in N$. Then $\left(\bar{x}_{k}\right) \in c^{i}(\Delta)$. Consider the sequence $\left(\bar{y}_{k}\right)$ defined by $\bar{y}_{k}=(-1)^{k}[k, k]$, for all $k \in N$. Then $\lim \Delta \bar{y}_{k}$ does not exists. We have $\left|\bar{y}_{k}\right| \leq\left|\bar{x}_{k}\right|$, for all $k \in N$. Hence $c^{i}(\Delta)$ is not solid and hence is not monotone. Similarly, for the others.

Theorem 3.5. The spaces $c_{0}^{i}(\Delta), c^{i}(\Delta)$ and $\ell_{\infty}^{i}(\Delta)$ are sequence algebra.

Proof: We prove that $c_{0}{ }^{i}(\Delta)$ is a sequence algebra. Let $\left(\bar{x}_{k}\right),\left(\bar{y}_{k}\right) \in$ $c_{0}^{i}(\Delta)$. Then $\lim _{k} \Delta \bar{x}_{k}=\theta$ and $\lim _{k} \Delta \bar{y}_{k}=\theta$ Then we have $\lim _{k} \Delta\left(\bar{x}_{k} \bar{y}_{k}\right)=\theta$.

Thus $\left(\bar{x}_{k} \bar{y}_{k}\right) \in c_{0}^{i}(\Delta)$ and hence a sequence algebra. For the space $c^{i}(\Delta)$ and $\ell_{\infty}^{i}(\Delta)$, the result can be proved similarly.

Theorem 3.6. The spaces $c^{i}(\Delta), c^{i}(\Delta)$ and $\ell_{\infty}^{i}(\Delta)$ are not convergence free in general.

Example 3.2. Let $\bar{x}_{k}=[k, k+1] \in c^{i}(\Delta)$ and $\bar{y}_{k}=\left[(-1)^{k}, 2+\frac{1}{K}\right]$ for all $(\mathrm{k} \in \mathrm{N})$. Then $\left(\bar{x}_{k}\right) \in c^{i}(\Delta)$ but $\left(\bar{y}_{k}\right) \in\left(c^{i}(\Delta)\right.$. Hence the space $c^{i}(\Delta)$ is not convergence free. Similarly, it can be proved that $c_{0}{ }^{i}(\Delta)$ is not convergence free.

Theorem 3.7. The inclusion $c_{0}^{i}(\Delta) \subset c^{i}(\Delta) \subset \ell_{\infty}^{i}(\Delta)$ holds and are strict.

Proof: The first inclusion follows from the definitions of the above classes of sequences.

The inclusion is strict follows from the following example.

It is obvious that $\bar{y}=([n, n+1]) \notin c_{0}^{i}(\Delta)$ but $\bar{y} \in c^{i}(\Delta)$. Since $y_{k_{l}}$ and $y_{k_{r}}$ are both divergent sequences and $\Delta(\bar{y})=\left(\Delta \overline{y_{k}}\right)=([k-k-2, k+1-$ $k-1])=([-2,0])$. Therefore, $\lim _{k} \Delta\left(y_{k_{l}}\right)=-2$ and $\lim _{k} \Delta\left(y_{k_{r}}\right)=0$. 


\section{References}

[1] A. Esi, Double lacunary Sequence Spaces of Double Sequence of Interval Numbers, Proyec. J. Math., 31(2), pp. 219-233, (2002).

[2] A. Esi, Lacunary Sequence Spaces of Interval Numbers,Thai J. Math., 10(2), pp. 445-451, (2012).

[3] A. J. Dutta; Class of $p$-absolutely summable sequences of interval numbers, New Trends in Analysis and Interdisiplinary Applications, Proc. 10th ISAAC Congress, (Springer), pp. 455-461, (2015).

[4] B. C. Tripathy and A Baruah, New type of difference sequence spaces of fuzzy real numbers, Math. Model. \& Anal., 14(3), pp. 391-397 (2009).

[5] B. C. Tripathy and M. Sen, On generalized statistically convergent sequences, Indian J. Pure Appl. Math., 32(11), pp. 1689-1694 (2001).

[6] B. C. Tripathy and S. Debnath, On generalized difference sequence spaces of fuzzy numbers, Acta Scientiarum. Technology, 35(1), pp. 117-121, (2013).

[7] H. Kizmaz, On certain sequence spaces, Canada. Math. Bull. 24, pp. 169-176, (1981).

[8] Kuo- Ping Chiao, Fundamental Properties of Interval Vector maxnorm, Tamsui Oxford J. Math. Sci., 18 (2), pp. 219-233, (2002).

[9] M. Et and R. Colak, On some generalized difference sequence spaces, Soochow J.of Math., 21, pp. 377-386 (1995).

[10] M. Sengonul and A. Eryilamz, On the Sequence Spaces of Interval Numbers, Thai J. Math., 8(3), pp. 503-510, (2010).

[11] P.K.Kamthan and M. Gupta, Sequence Spaces and Series, Marcel Dekker, (1981).

[12] P. S. Dwyer, Linear Computation, New York, Wiley, (1951).

[13] P. S. Dwyer, Erros of Matrix Computation, Simultaneous Equations and Eigenvalues, National Bureu of Standarts, Applied Mathematics Series, 29, pp. 49-58, (1953). 
[14] P. S. Fischer, Automatic Propagated and Round-off Error Analysis, Paper presented at the 13th national meeting of the Association for Computing Machinary, June (1958).

[15] R. E. Moore, Automatic Error Analysis in Digital Computation, LSMD-48421, Lockheed Missiles and Space Company, (1959).

[16] R. E. Moore and C. T. Yang, Theory of an Interval Algebra and Its Application to Numeric Analysis, RAAG Memories II, Gaukutsu Bunken Fukeyu-kai, Tokyo, (1962).

[17] S. Debnath, B. Sarma and S. Saha, On some sequence spaces of interval vectors, Afr. Math., 26 (5), pp. 673-678, (2015).

[18] S. Debnath, A. J. Dutta and S. Saha, Regular matrix of interval numbers based on Fibonacci numbers, Afr. Math., 26(7), pp. 1379-1385, (2015).

[19] S.Debnath and S. Saha, On Statistically Convergent Sequence Spaces of Interval Numbers, Proceedings of IMBICV-(3), pp. 178-182, (2014) .(ISSN-978-81-925832-28).

[20] S.Debnath and S. Saha, Some Statistically Convergent Difference Sequence Spaces of Interval Numbers,ROMAI J., 12(1), pp. 53-59, (2016).

[21] S.Debnath and S. Saha, On Statistically Convergent Sequence Spaces of Two Dimensional Interval Vectors, J. Indian Academy of Mathematics, 38(1), pp. 29-37, (2016).

[22] S.Debnath and S. Saha, Matrix Transformation on Statistically Convergent Sequence Spaces of Interval Number Sequences, Proec. J Math., 35 (1), pp. 187-195, (2016).

\author{
Binod Chandra Tripathy \\ Department of Mathematics \\ Tripura University \\ Suryamaninagar-799022 \\ Agartala \\ India \\ e-mail : binodtripathy@tripurauniv.in
}

\title{
Shyamal Debnath
}


612 Binod Chandra Tripathy, Shyamal Debnath and Subrata Saha

Department of Mathematics

Tripura University

Suryamaninagar-799022

Agartala

India

e-mail : debnathshyamal@tripurauniv.in

and

Subrata Saha

Department of Mathematics

Tripura University

Suryamaninagar-799022

Agartala

India

e-mail : subratasaha@gmail.com 